\section{KOMPASS}

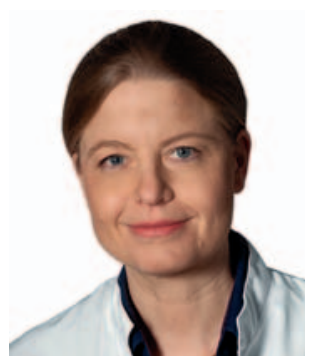

Karin Jordan

Innere Medizin, Universitätsklinikum

Heidelberg, Deutschland

Eine effektive und für den Patienten verträgliche Tumortherapie ist ohne eine individuell durchdachte und umgesetzte Supportivtherapie nicht denkbar. Der Wert der Supportivtherapie wird dabei in prägnanter Weise durch den Leitsatz der MASCC (Multinational Association of Supportive Care in Cancer) verdeutlicht: «Supportive care makes excellent cancer care possible».

Im vergangenen Jahr wurde die S3-Leitlinie «Supportive Therapie bei onkologischen Patienten» [1] mit dem Ziel veröffentlicht, eine deutschlandweite Standardisierung bei wichtigen Themen der onkologischen Supportivtherapie zu erreichen. Formal konsentierte und klar verständliche Empfehlungen sollen dabei sowohl Ärzte und andere Berufsgruppen als auch die betroffenen Patienten bei medizinischen Entscheidungen unterstützen. Die Leitlinie entstand unter der Federführung der Arbeitsgemeinschaft «Supportive Maßnahmen in der Onkologie, Rehabilitation und Sozialmedizin» (ASORS) in der Deutschen Krebsgesellschaft, der Deutschen Gesellschaft für Hämatologie und Medizinische Onkologie (DGHO) und der Deutschen Gesellschaft für Radioonkologie (DEGRO) und wurde am Universitätsklinikum Halle (Saale) koordiniert. Diese erste organunabhängige Querschnittsleitlinie thematisiert zehn Bereiche der Supportivtherapie: Tumortherapie-induzierte Anämie, Neutropenie, Nausea und Emesis, Diarrhoe, orale Mucositis, Hauttoxizität, periphere Neuro-

\title{
Supportivtherapie - ein unverzichtbarer Baustein der onkologischen Behandlung
}

toxizität, ossäre Komplikationen, Paravasate und supportive Maßnahmen in der Radioonkologie. Zwar umfasst das Spektrum der supportiven Therapie eine weit größere Anzahl wichtiger Themen, jedoch ist es ressourcenbedingt aktuell nicht möglich, ein jedes dieser weitgefächerten Themen in nur einer Leitlinie umfassend und befriedigend darzustellen.

Der Startschuss für den Beginn der Leitlinienarbeit fiel bereits im März 2013 mit dem Kick-off Meeting, bei dem alle 88 Mandatsträger bzw. Experten der 45 beteiligten Fachgesellschaften, Arbeitsgemeinschaften und Patientengruppen die insgesamt 120 Schlüsselfragen der zehn Einzelthemen konsentierten. In zwei Konsensustreffen wurden die insgesamt 337 Empfehlungen bzw. Statements abgestimmt. Am Ende steht als Ergebnis eine 558 Seiten füllende Langversion der S3-Leitlinie, ein 148 Seiten langer Leitlinienreport, 626 Seiten Evidenztabellen und eine Patientenleitlinie, an der zurzeit noch gearbeitet wird. Der sachgerechte Einsatz der supportiven Therapie in der Krebsbehandlung ist ein wesentliches Qualitätsmerkmal für eine onkologische Behandlung - das gilt für Patienten aller Altersgruppen von der Diagnosestellung und Erstbehandlung bis hin zur palliativen Versorgung. Mit der Veröffentlichung dieser Leitlinie erhoffen wir uns, dass die supportive Therapie als wesentlicher Bestandteil jeglicher Behandlung onkologischer Erkrankungen stärker in den Fokus der Krebstherapie rückt und weniger als ein Kann, sondern mehr als ein Muss verstanden wird, um die Therapie für den Patienten verträglicher zu machen, die Fortführung der geplanten Tumortherapie zu sichern und zu einer guten bzw. akzeptablen Lebensqualität der Patienten beizutragen.

In dieser Ausgabe von Karger Kompass ONKologie finden Sie zahlreiche Beispiele zur Umsetzung der Leitlinie in der Praxis. Zudem bilden die Abstracts des 5. ASORSJahreskongresses «Supportive Therapie und Rehabilitation bei Krebs, State of the Art 2017» [2] das breite Spektrum dieses sehr lebendigen und vielschichtigen Forschungsgebietes ab. Konkrete Hinweise zu supportivmedizinischen Maßnahmen sind auch in den Steckbriefen der Therapieschemata FOLFOX und FOLFIRI enthalten, die Sie als Beilage zur Rubrik «Campus» in diesem Heft finden.

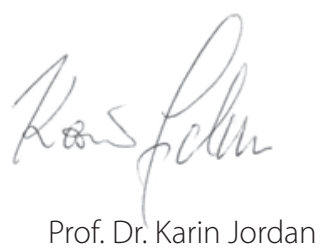

\section{Literatur}

1 Leitlinienprogramm Onkologie: S3-Leitlinie Supportive Therapie bei onkologischen Patient Innen. www.leitlinienprogramm-onkologie.de/ Supportive-Therapie.95.0.html (Zugriff: 28.02. 2017).

2 Supportive Therapie und Rehabilitation bei Krebs, State of the Art 2017. Oncol Res Treat 2017;40(suppl 1):1-19. www.karger.com/Journal/ Issue/275841.

\section{KARGER}

Fax +49761 4520714

information@karger.com

www.karger.com
() 2017 S. Karger GmbH, Freiburg

Accessible online at: www.karger.com/kko
Prof. Dr. Karin Jordan

Innere Medizin V

Universitätsklinikum Heidelberg

Im Neuenheimer Feld 410, 69120 Heidelberg, Deutschland

karin.jordan@med.uni-heidelberg.de 